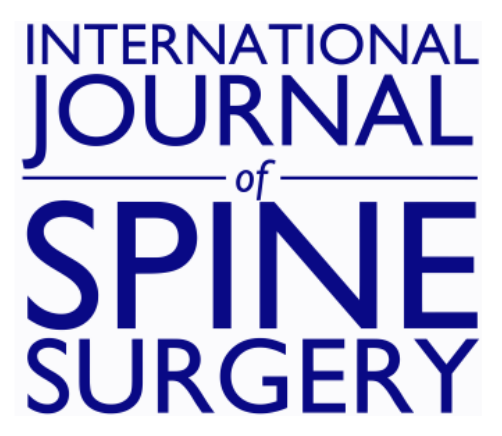

\title{
A Novel Endoscopic Technique for Biopsy and Tissue Diagnosis for a Paraspinal Thoracic Tumor in a Pediatric Patient: A Case Report
}

Sanjay Konakondla, Jonathan Nakhla, Jimmy Xia, Sean M. Barber, Jared S. Fridley, Adetokunbo A. Oyelese, Ziya L. Gokaslan, Nikolai G. Rainov, Dimitar G. Haritonov, Ralf Wagner and Albert E. Telfeian

Int J Spine Surg 2021, 14 (s4) S66-S70

doi: https://doi.org/10.14444/7167

http://ijssurgery.com/content/14/s4/S66

This information is current as of April 26, 2023.

Email Alerts Receive free email-alerts when new articles cite this article. Sign up at:

http://ijssurgery.com/alerts

The International Journal of Spine Surgery

2397 Waterbury Circle, Suite 1,

Aurora, IL 60504, Phone: +1-630-375-1432 


\title{
A Novel Endoscopic Technique for Biopsy and Tissue Diagnosis for a Paraspinal Thoracic Tumor in a Pediatric Patient: A Case Report
}

SANJAY KONAKONDLA, MD, ${ }^{1}$ JONATHAN NAKHLA, MD,${ }^{1}$ JIMMY XIA, BSC, ${ }^{1}$ SEAN M. BARBER, MD, ${ }^{1}$ JARED S. FRIDLEY, MD ${ }^{1}$ ADETOKUNBO A. OYELESE, MD, PHD,${ }^{1}$ ZIYA L. GOKASLAN, MD, ${ }^{1}$ NIKOLAI G. RAINOV, MD,${ }^{2}$ DIMITAR G. HARITONOV, MD, PHD,${ }^{3}$ RALF WAGNER, $\mathrm{MD},{ }^{4}$ ALBERT E. TELFEIAN, $\mathrm{MD}, \mathrm{PHD}^{1}$

${ }^{I}$ Warren Alpert Medical School of Brown University, Rhode Island Hospital, Providence, RI, ${ }^{2}$ MVZ Wirbelsäulenzentrum Taufkirchen b. München, Munich, Germany, ${ }^{3}$ University Hospital Heart and Brain, Pleven, Bulgaria, ${ }^{4}$ Ligamenta Spine Centre, Frankfurt am Main, Germany

\begin{abstract}
Background: Conventional approaches to the thoracic spine can require extensive tissue dissection, bony disruption, and instability that may warrant the need for instrumentation and fusion. Furthermore, anterior approaches may require the involvement of various surgeons from multiple disciplines to ensure a successful operation and mitigate complications. Currently, available minimally invasive approaches still require bony removal and usually rely heavily on computed tomography (CT)-guided imaging without direct gross visualization. Endoscopic spinal procedures have provided an ultra-minimally invasive alternative to access many areas in and around the spinal column.

Methods: We present a 12-year-old boy with a right-sided $2.0 \times 3.2-\mathrm{cm}$ paravertebral lesion at the level of T5. The patient successfully underwent an endoscopic approach to the lesion with minimal tissue and bony disruption for tissue diagnosis and tumor resection.

Results: At initial and 6-month follow-up, the patient remained asymptomatic and without issues.

Conclusions: We demonstrate here the feasibility and suggest the safety of a posterior ultra-minimally invasive endoscopic spinal approach to obtain a tissue biopsy of an incidentally found ventrolateral paraspinal tumor in the thoracic region in a pediatric patient. This minimal approach can prove to achieve similar results as other approaches that may otherwise necessitate more extensive or transthoracic procedures.

Minimally Invasive Surgery

Keywords: endoscopic spine surgery, thoracic tumor, paraganglioma
\end{abstract}

\section{INTRODUCTION}

Access to paraspinal tumors in the thoracic region requires thorough planning and discussion to select the most suitable approach. Generally, the available approaches can be categorized into posterior, anterior, lateral, or a combination of these approaches. Furthermore, we can subclassify these approaches into open or minimally invasive. Open posterior approaches to paraspinal tumors in the thoracic region often demand a wide exposure and significant muscle disruption and require bony removal of the posterior spinal elements with partial removal of unilateral or bilateral joints and ribs. Extensive bony removal can cause concern for iatrogenic spinal instability, which may necessitate the need for a more involved operation requiring instrumentation and fusion. Open anterior or lateral transthoracic approaches can frequently require the involvement of multiple surgeons with expertise in various disciplines for both approach and tumor resection. ${ }^{1}$ Minimally invasive or computed tomography (CT)-guided approaches to these areas can be transpedicular posteriorly or transthoracic from an anterior or lateral approach, though these also often involve extensive bony removal, poor visualization, additional radiation, and tumor contamination, among other limitations. ${ }^{2}$ These CT-guided needle approaches, moreover, do not allow for maximally safe tumor resection.

As advancements in endoscopic equipment and visibility progress and evolve, the indications for endoscopic techniques to treat various spinal pathologies are rapidly increasing. ${ }^{3,4}$ The requirement of precise localization, nuanced operative techniques, and familiarization of surgical anatomy, 

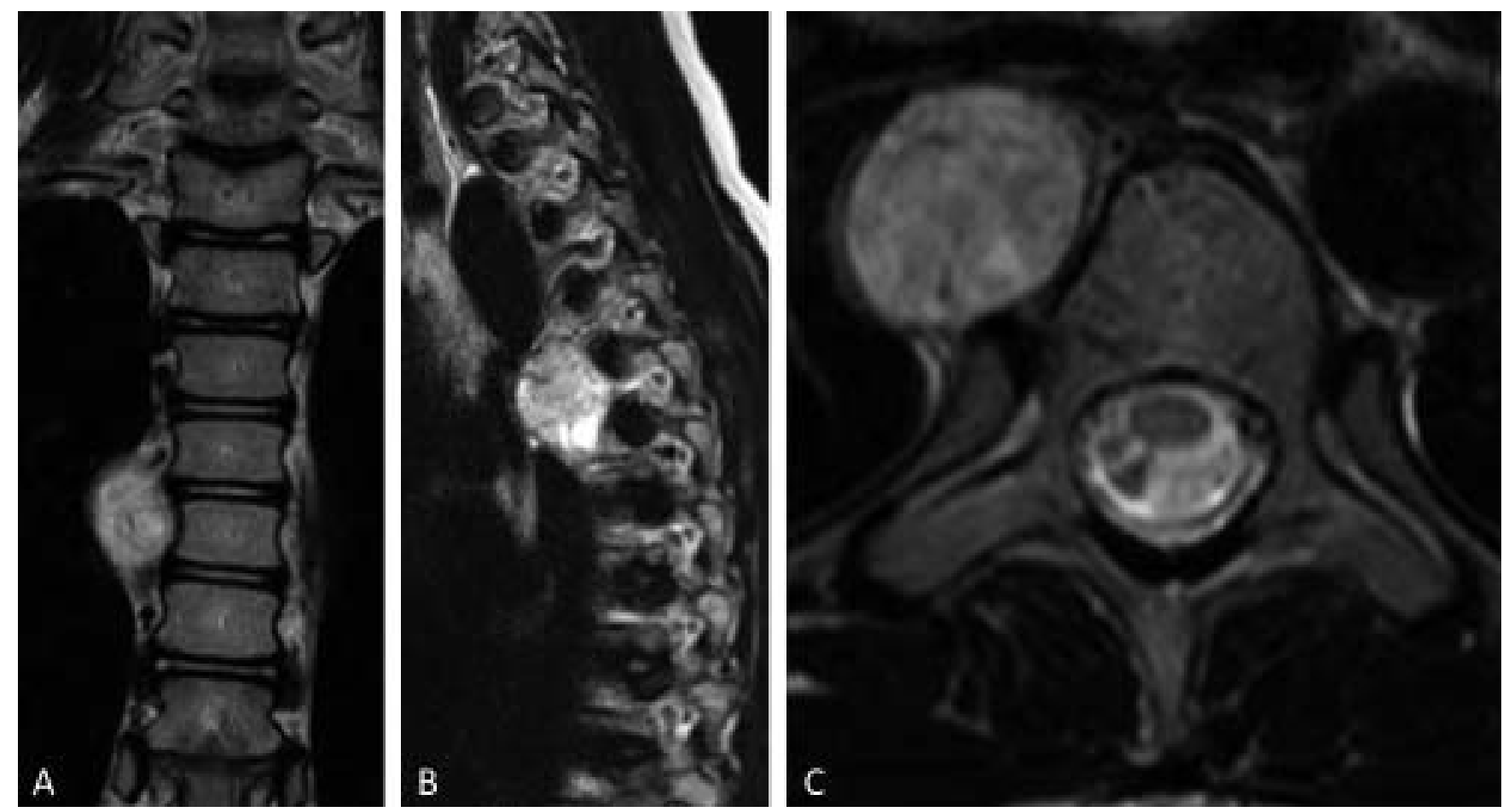

Figure 1. MRI findings. T2 weighted sequences revealing a paraspinal enhancing lesion at the level of T5 (A: coronal plane; $\mathrm{B}$ : sagittal plane), ventral to the costovertebral joint on the right side $(C)$.

however, makes endoscopic spine procedures uniquely challenging. Herein we describe the application of an endoscopic spine technique for tissue biopsy and diagnosis of a paraspinal thoracic tumor in a pediatric patient that avoids all issues of conventional approaches.

\section{METHODS}

\section{Case Report}

The patient is a 12-year-old boy with a history of a T5-T10 spinal cord arteriovenous malformation status postembolization. At the time of follow-up, a surveillance MRI was completed that incidentally

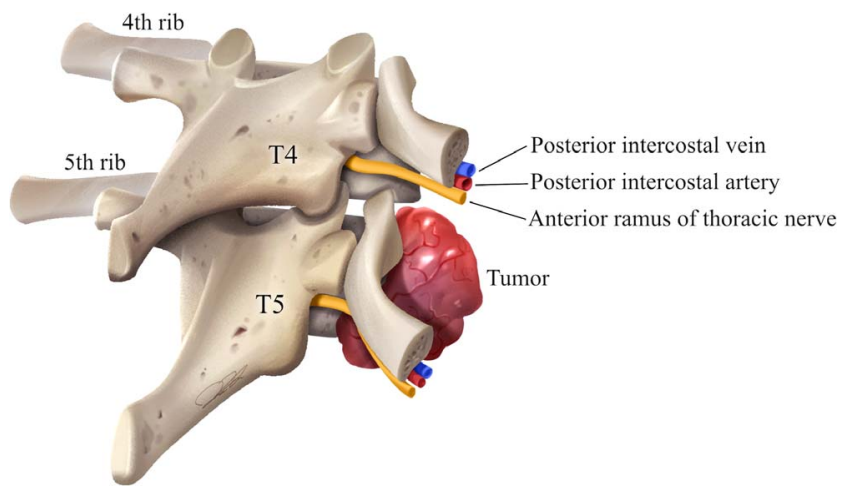

Figure 2. Tumor location. Artist representation of right ventrolateral tumor at the costovertebral joint at the level of T5. revealed a right-sided $2.0 \times 3.2-\mathrm{cm}$ paravertebral lesion at the level of T5 with moderate enhancement with gadolinium (Figures 1 and 2). The patient did not have any complaints or symptoms localizing to this lesion and was neurologically intact. The decision was made to obtain a tissue specimen to establish a diagnosis.

\section{Endoscopic Operative Technique and Localization}

The procedure was performed after the patient was induced under general endotracheal anesthesia without complication. The patient was placed in a prone position onto a Wilson frame. All pressure points were accounted for and padded appropriately. Fluoroscopic imaging was used for level localization and precise targeting. The incision was planned by measuring $3 \mathrm{~cm}$ lateral to the spinous process of $\mathrm{T} 4$ on the right side. Local anesthetic was given prior to incision. A spinal needle was navigated to the costovertebral joint at T5. Dilators were navigated and advanced sequentially to this target to facilitate placement of the endoscopic working channel (Figure 3A). A $6.8-\mathrm{mm}, 30^{\circ}$ TESSYS Joimax (Karlsruhe, Germany) endoscope was subsequently passed through the working channel. The tumor was immediately visualized upon entry, and multiple samples could be obtained 

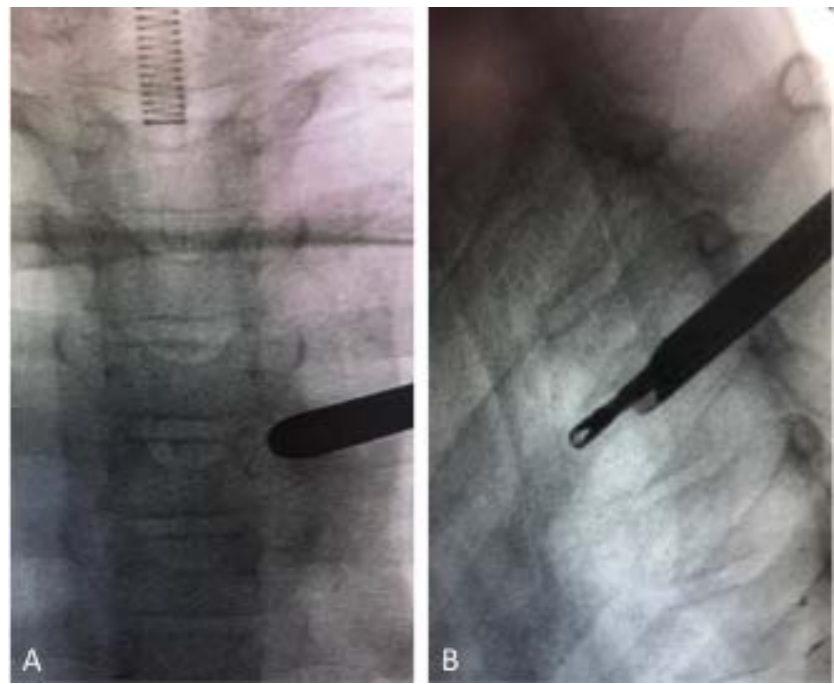

Figure 3. Working channel. (A) AP projection of the working channel aiming towards the right T5 costovertebral joint directly onto the tumor with $3.5-\mathrm{mm}$ Joimax grasper forceps used to obtain the specimen shown in the lateral projection (B). AP, anteroposterior.

with 3.5-mm Joimax grasper forceps (Figures 3B and 4). Hemostasis was obtained with both bipolar electrocautery under direct visualization and free surface hydraulics with irrigation. Joimax grasper forceps were used to continue with tumor resection, and hemostasis techniques were applied as necessary until adequate tumor was removed. The safety of the tumor resection was based on the tumor's proximity to the superior vena cava.

\section{Diagnosis and Postoperative Course}

The specimen histologically and immunohistochemically revealed a lobular architecture containing giant cells with abundant bright eosinophilic cytoplasm. A characteristic Zellballen structure was demonstrated (Figure 5A). The nuclei were found with mild polymorphism with chromatin and anastomosing blood vessels. Pan cytokertin stains were mildly positive and S100 was positive in the supporting cells making up the lobular structure. The tumor was CD30 negative and CD117 positive. Synaptophysin and chromogranin A were diffusely positive (Figure 5B and 5C). The Ki-67 index was $4.8 \%$. Final pathological diagnosis was noted to be paraganglioma. The patient did not have any family history of paraganglioma and genetic testing was pursued. Immediate postoperative MRI was completed during the initial hospital stay to assess resection and to aid in surveillance. Significant tumor resection was noted with expected residual along the superior vena cava. The patient did well

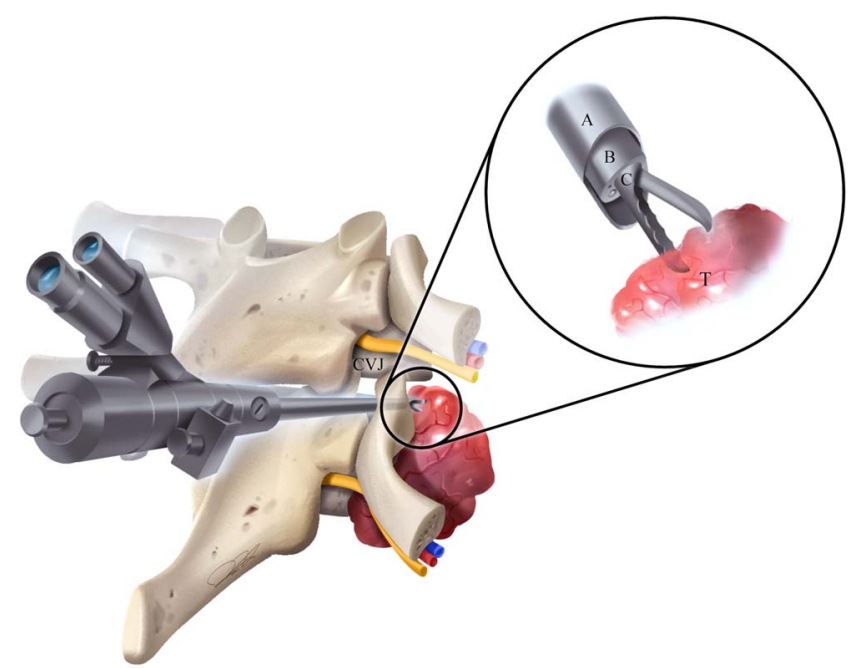

Figure 4. Surgical technique. Artist representation of the surgical approach from a paravertebral incision through the T5 costovertebral joint (CVJ) to biopsy the right ventrolateral tumor $(\mathrm{T})$ using $(\mathrm{C})$ grasper forceps $(\mathrm{B})$ through the endoscope via (A) a working channel.

and was discharged on the third postoperative day without any issues or complications. On the first postoperative follow-up visit, the patient was asymptomatic, pain free, and without complications. At 7 months, the patient was asymptomatic without any issues. Follow-up MRI at this time was completed for surveillance and did not reveal any tumor recurrence or growth.

\section{DISCUSSION}

Spinal paragangliomas are rare, slow-growing, potentially secretory tumors that rarely involve the spine. These tumors are even more rare in the thoracic region, which is evidenced by case reports of paraspinal thoracic paragangliomas. ${ }^{1,5}$ The definitive diagnosis of spinal paragangliomas is made upon histopathological examination. ${ }^{6}$ Due to nonspecific radiographic findings, preoperative differential diagnosis of paraspinal tumors must include schwannoma, neurofibroma, and malignant nerve sheath tumors as well as paraganglioma. Though classified by the World Health Organization as grade I benign tumors, malignant transformation has been reported in the literature up to $14 \%{ }^{6}$ Primary treatment involves complete resection to prevent recurrence and improve progression-free survival.

Along with tumor size and location, tissue diagnosis can assist with subsequent surgical planning and multidisciplinary discussions. Various approaches for the complete resection of paraspinal tumors of the thoracic spine have been described. ${ }^{7}$ 

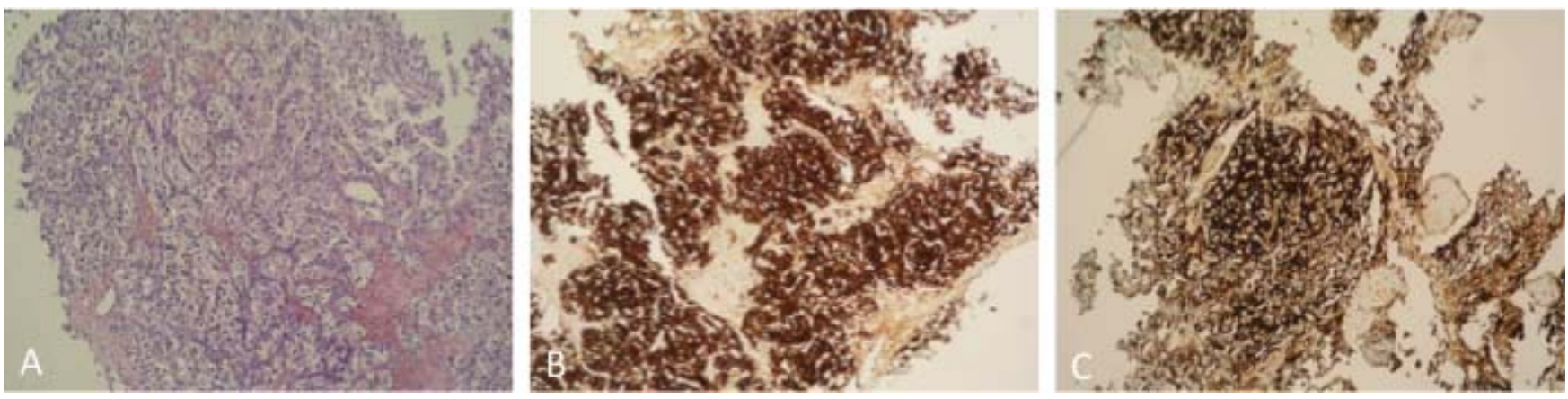

Figure 5. Paraganglioma. (A) Cytology revealing Zellballen structure with (B) positive synaptophysin and (C) chromogranin A, 100× magnification.

Minimally invasive techniques to access intradural lesions in the thoracic spine have been described, though traditional techniques to access the paraspinal thoracic region require both a different approach and more bony removal. ${ }^{8}$ Dumbbell tumors resected through minimally invasive approaches may still warrant bony removal and fusion. ${ }^{9}$ These open and minimally invasive techniques, however, have been primarily used only for tumor resection rather than for obtaining tissue for diagnosis, because these approaches demand more extensive exposures and arduous procedures.

CT-guided biopsy techniques are feasible alternatives to an endoscopic biopsy to access lesions in this area. The advantage of this technique allows for high-quality CT images and identification of bony landmarks for needle guidance. Disadvantages of CT-guided techniques include the lack of real-time needle passing, radiation exposure, tumor contamination, and high cost. ${ }^{2}$ With the endoscopic biopsy, the tissue is under direct visualization throughout the procedure on a high-definition screen. Fluoroscopic guidance can be used sparingly to minimize radiation exposure during localization. Traditional biopsy techniques can create concern for seeding tumor cells along the biopsy tract. Because of these concerns, with biopsies there can be significant clinical relevance and can have implications on future surgical planning for complete tumor resection. With the endoscopic biopsy, a tissue dilator is used to facilitate the use of a working channel. This may help mitigate contamination concerns because the surrounding tissue along the tract is protected. Though the concern of sampling error in all biopsy techniques exists, the larger working channel also allows maneuvering to create a larger surface area for more potential regions to biopsy. After the biopsies are taken under direct endoscopic visualization, areas can be inspected for obvious bleeders.
Hemostasis can be immediately and effectively obtained with certainty, which is a unique advantage to the endoscopic technique compared with conventional image-guided biopsy techniques.

Ultra-minimally invasive endoscopic techniques gain entry to the areas of interest by accessing natural corridors such as the Kambin triangle, for example, during transforaminal endoscopic discectomies. ${ }^{10}$ Studies have also shown favorable complication rates with endoscopic spine surgery when compared with minimally invasive or open spine surgeries. ${ }^{11}$ In a multi-institutional study comprising 553 consecutive patients, the rate of intraoperative and postoperative complications was reported to be $2.7 \%$. Complications reported included durotomies, epidural hematomas, recurrent disc herniations, and systemic complications.

Endoscopic procedures have been described for the treatment of thoracic discs ${ }^{12}$ and epidural thoracic lesions $^{13,14}$; however, these were all completed using a transforaminal technique to access the neural foramen and the epidural space. To conclusively demonstrate safety, the outcomes of more cases should be reviewed, and perhaps, cadaveric studies may be completed to assess postprocedural collateral damage and complications. Herein we describe the first totally posterior paraspinal approach for targeting, biopsy, and tissue diagnosis of a ventrolateral paraspinal thoracic tumor.

\section{CONCLUSION}

Ultra-minimally invasive endoscopic spinal surgery from a posterior approach may be a safe alternative for tissue biopsy and histological diagnosis of paraspinal lesions, which may otherwise necessitate more extensive or transthoracic procedures. 


\section{REFERENCES}

1. Nawaz MA, Samarage M, O’Neill K, Punjabi P. Management of a giant thoracic hypervascular paraspinal ganglioma. Ann Thorac Surg. 2012;93(1):e7-e8. doi:10.1016/j. athoracsur.2011.07.060

2. Kalhan S, Sharma P, Sharma S, Dudani S, Ramakrishnan T, Chowdhry A. Evaluation of precision of guidance techniques in image guided fine needle aspiration cytology of thoracic mass lesions. J Cytol. 2012;29(1):6-10. doi:10.4103/0970-9371.93209

3. Telfeian AE, Oyelese A, Fridley J, Gokaslan ZL. Transforaminal endoscopic decompression for foot drop 12 years after lumbar total disk replacement. World Neurosurg. 2018;116:136-139. doi:10.1016/j.wneu.2018.05.089

4. Telfeian AE. An awake, minimally invasive, fully endoscopic surgical technique for treating lumbar radiculopathy secondary to heterotopic foraminal bone formation after a minimally invasive transforaminal lumbar interbody fusion with BMP: technical note. J Spine Surg (Hong Kong). 2018;4(1):162-166. doi:10.21037/jss.2018.03.08

5. Akhtar S, Sattar S, Bari E, Kayani N, Moeen S. Secretory paraspinal paraganglioma of thoracolumbar spine: case report and review of literature. Surg Neurol Int. 2016;7(suppl 37):S889-S892. doi:10.4103/2152-7806.194510

6. Turk O, Yaldiz C, Antar V, et al. Spinal paragangliomas: surgical treatment and follow-up outcomes in eight cases. Medicine (Baltimore). 2018;97(38):e12468. doi:10.1097/MD. 0000000000012468

7. Rong H-T, Fan Y-S, Li S-P, et al. Management of dumbbell and paraspinal tumors of the thoracic spine using a single-stage posterolateral approach: case series. Orthop Surg. 2018;10(4):343-349. doi:10.1111/os.12405

8. Tumialán LM, Theodore $\mathrm{N}$, Narayanan M, Marciano FF, Nakaji P. Anatomic basis for minimally invasive resection of intradural extramedullary lesions in thoracic spine. World Neurosurg. 2018;109:e770-e777. doi:10.1016/j.wneu.2017.10. 078

9. Li C, Ye Y, Gu Y, Dong J. Minimally invasive resection of extradural dumbbell tumors of thoracic spine: surgical techniques and literature review. Eur Spine J. 2016;25(12):41084115. doi:10.1007/s00586-016-4677-z

10. Hardenbrook M, Lombardo S, Wilson MC, Telfeian
AE. The anatomic rationale for transforaminal endoscopic interbody fusion: a cadaveric analysis. Neurosurg Focus. 2016;40(2):E12. doi:10.3171/2015.10.FOCUS15389

11. Sen RD, White-Dzuro G, Ruzevick J, et al. Intra- and perioperative complications associated with endoscopic spine surgery: a multi-institutional study. World Neurosurg. 2018;120:e1054-e1060. doi:10.1016/j.wneu.2018.09.009

12. Wagner R, Telfeian AE, Iprenburg M, et al. Transforaminal endoscopic foraminoplasty and discectomy for the treatment of a thoracic disc herniation. World Neurosurg. 2016;90:194-198. doi:10.1016/j.wneu.2016.02.086

13. Telfeian AE, Choi DB, Aghion DM. Transforaminal endoscopic surgery under local analgesia for ventral epidural thoracic spinal tumor: case report. Clin Neurol Neurosurg. 2015;134:1-3. doi:10.1016/j.clineuro.2015.03.022

14. Joo Y-C, Ok W-K, Baik S-H, Kim H-J, Kwon O-S, Kim $\mathrm{K}-\mathrm{H}$. Removal of a vertebral metastatic tumor compressing the spinal nerve roots via a single-port, transforaminal, endoscopic approach under monitored anesthesia care. Pain Physician. 15(4):297-302. http://www.ncbi.nlm.nih.gov/pubmed/22828683

Disclosures and COI: The authors received no funding for this study and report no conflicts of interest.

Corresponding Author: Albert E. Telfeian, MD, PhD, Department of Neurosurgery, Norman Prince Neurosciences Institute, Warren Alpert Medical School of Brown University, Rhode Island Hospital, 593 Eddy St, Providence, RI 02903. Phone: (401) 793-9132; Email: atelfeian@Lifespan. org.

Published 11 February 2021

This manuscript is generously published free of charge by ISASS, the International Society for the Advancement of Spine Surgery. Copyright $(\subset 2020$ ISASS. To see more or order reprints or permissions, see http://ijssurgery.com. 